\title{
Rheumatologic manifestations of hepatic diseases
}

\author{
Bruno Fernandes ${ }^{a *}$, Emanuel Dias ${ }^{b *}$, Miguel Mascarenhas-Saraiva ${ }^{b}$, Miguel Bernardes $^{a}$, Lúcia Costa ${ }^{a}$, \\ Hélder Cardoso ${ }^{b}$, Guilherme Macedo ${ }^{b}$
}

Centro Hospitalar de São João, Porto, Portugal

\section{Abstract}

The course of hepatic diseases may be complicated by a multitude of rheumatologic manifestations, which can complicate the diagnostic approach and alter the natural history of primary liver disease, sometimes worsening prognosis due to associated multiple organ dysfunction. These manifestations can occur in association with a multitude of liver diseases, including viral hepatitis, autoimmune hepatitis, alcoholic liver disease, nonalcoholic fatty liver disease, hemochromatosis, or Wilson's disease. It is necessary not only for rheumatologists, but also for other clinicians, to be aware that these atypical manifestations may reflect an undiagnosed hepatic disease. On the other hand, it is crucial that, in a patient with known hepatic disease presenting with rheumatologic symptoms, an accurate distinction be made between the rheumatologic manifestations of hepatic disease and primary rheumatologic disease, since the treatment is often different. This review aims to summarize the current evidence regarding rheumatologic manifestations of hepatic diseases, how to distinguish them from primary rheumatologic disorders, and how to provide adequate management.

Keywords Rheumatologic manifestations, arthritis, hepatic diseases, hepatitis

\section{Introduction}

Rheumatologic manifestations of gastrointestinal diseases have been known for decades [1]. As long as new studies are published, it is possible not only to understand their pathophysiology but also to unravel new rheumatologic manifestations previously unknown. In fact, there are myriad gastrointestinal diseases that can affect the musculoskeletal system in multiple ways.

Hepatic diseases are particularly relevant, since the liver plays an important role in mediating immune mechanisms, which currently represent the strongest hypothesis to explain the pathophysiology of their rheumatologic manifestations. This review focuses on the rheumatologic manifestations

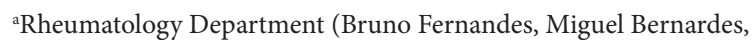

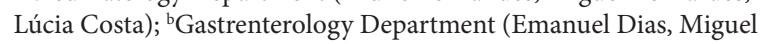
Mascarenhas-Saraiva, Hélder Cardoso, Guilherme Macedo), Centro Hospitalar de São João, Porto, Portugal

${ }^{\star}$ These authors contributed equally

Conflict of Interest: None

Correspondence to: Emanuel Dias, MD, Gastroenterology Department, Centro Hospitalar de São João, Alameda Professor Hernâni Monteiro, PT - 4200-319, Porto, Portugal, e-mail: diasj0310@gmail.com

Received 26 December 2018; accepted 1 April 2019; published online 20 May 2019

DOI: https://doi.org/10.20524/aog.2019.0386 of hepatic disease [2], especially hepatitis C, a liver disease currently viewed as systemic, where the involvement of joints considerably affects prognosis and quality of life [3]. Nevertheless, many other liver diseases may be associated with joint involvement. The aim of this review is to synthesize the most extensively studied rheumatologic manifestations of liver diseases to date, highlighting the importance of distinguishing these from primary rheumatologic diseases, since their management and outcome are largely affected by an accurate diagnosis.

\section{Hepatitis C}

\section{General considerations}

Hepatitis C virus (HCV) infection is a global health problem, affecting approximately 20 million people across Europe and 170 million people worldwide, and causing chronic hepatitis, cirrhosis and hepatocellular carcinoma, with liver-related mortality in about 350,000 people/year [4]. Nevertheless, beyond the classic hepatic complications, HCV infection is currently viewed as a systemic disease. In fact, during $\mathrm{HCV}$ chronic infection, extrahepatic manifestations are frequent and polymorphous, occurring in about two thirds of infected people and involving primarily the joints, muscles and skin. In fact, rheumatologic manifestations are the most common extrahepatic manifestation of hepatitis $\mathrm{C}$ and their 
incidence is variable according to geographic area, but can reach $38 \%$ (Table 1) [5].

Autoimmune and lymphoproliferative diseases are well-studied examples of $\mathrm{HCV}$-associated diseases. Although the pathophysiology of many of these diseases is still obscure, it can be at least partially explained by the fact that HCV has double cellular tropism, being not only hepatotropic but also lymphotropic [6].

$\mathrm{HCV}$ eradication is often associated with improvement of extrahepatic symptoms. In this regard, the emergence of directacting antiviral agents offers the opportunity to treat both HCV infection and its systemic manifestations, requiring shorter treatment duration and driving minor adverse effects [7].

\section{Mixed cryoglobulinemia}

The association between mixed cryoglobulinemia and HCV infection is well established [8], with hepatitis $C$ representing the cause in about $80 \%$ of cases [9]. This is a predominantly small-vessel systemic vasculitis, characterized by the presence of serum immunoglobulins that precipitate reversibly at temperatures lower than $37^{\circ} \mathrm{C}$ and solubilize again when the temperature rises. Three distinct types of cryoglobulins have been recognized. In type I, the immunoglobulins are usually monoclonal and associated with lymphoproliferative diseases. Type II primarily consists of IgM rheumatoid factor and polyclonal IgG; this type is usually associated with viral infections and inflammatory diseases and is induced by HCV in up to $60 \%$ of cases. Type III cryoglobulins are mixed polyclonal and there is no monoclonal proliferation. Mixed cryoglobulinemias include type II and III. Cryoglobulins are present in the serum of $40-60 \%$ of patients with $\mathrm{HCV}$, although disease occurs in only $5 \%$ (usually women aged more than 50 years) $[3,10]$.

The relation between mixed cryoglobulinemia and hepatitis Chas been clearly demonstrated:HCV-RNA sequences have been found both in cryoprecipitates and in cutaneous lesions of patients with mixed cryoglobulinemia [11]. The pathophysiology of this association is, similarly to other $\mathrm{HCV}$ extrahepatic manifestations, probably related to activation of B-cell lymphocytes with subsequent production of autoantibodies and mixed cryoglobulins [12]. It is important to highlight that, despite the fact that there is no correlation between mixed cryoglobulinemia and HCV genotype, there is an important epidemiological association between mixed cryoglobulinemia and severe hepatic disease [13]. According to its pathophysiology, mixed cryoglobulinemia may be

Table 1 Rheumatologic diseases associated with hepatitis C, according to their degree of association

\begin{tabular}{lll}
\hline High & Medium & Low \\
\hline $\begin{array}{l}\text { Mixed } \\
\text { cryoglobulinemia }\end{array}$ & Sicca syndrome & Sjögren's syndrome \\
& $\begin{array}{l}\text { Arthritis } \\
\text { Osteosclerosis }\end{array}$ & Polyarteritis nodosa \\
\hline
\end{tabular}

seen not only as an autoimmune disease, but also as a B-cell lymphoproliferative disease, which can explain malignant transformation in $8-10 \%$ of cases and a well-established association with non-Hodgkin B-cell lymphoma [14].

Although classically described by the triad of purpura, fatigue and arthralgia, the various involvement of different organs and tissues (mainly skin, joints, kidney, peripheral nerves) leads to variable clinical presentation and evolution. Polyarthralgia occurs in about $70 \%$ of cases and can recur intermittently throughout the course of illness. The most frequently involved joints include hands and knees in $45 \%$ of cases, followed by ankles and elbows in $25 \%$. Overt arthritis is rare ( $8 \%$ of cases), but when present it usually involves large joints, without erosion, even after several years. Skin is the most commonly affected organ and some studies indicate that cutaneous involvement is present in virtually $100 \%$ of cases: episodes of vascular purpura are the most common presentation, but patients can also present skin ulcers, Raynaud phenomenon and acrocyanosis. Sicca syndrome is described in about $30 \%$ of cases and renal involvement in about $20 \%$, most commonly type 1 membranoproliferative glomerulonephritis [10]. It may have an acute or chronic presentation, presents with proteinuria, hematuria and variable degrees of renal failure and is associated with IgM kappa type 2 mixed cryoglobulinemia [15]. Neurological involvement may also occur, with sensitive or motor-sensitive polyneuropathy. As a systemic disorder, other manifestations, like cardiomyopathy or intestinal ischemia, may also occur, though less commonly. Various attempts at defining a clinical classification have been made and, recently, preliminary classification criteria were developed according to the results of an international European study [16] and subsequently validated [17].

Among $\mathrm{HCV}$-infected patients, the predictive factors most strongly associated with mixed cryoglobulinemia are advanced age, long duration of disease, type II mixed cryoglobulins, a higher mixed cryoglobulin serum level, and B-cell clonal expansion. Mortality is variable, ranging between $20 \%$ and $80 \%$, and depends on involved organ systems [18]. Advanced age by the time of diagnosis and renal involvement are predictive of poor outcome [19]. Intestinal ischemia, pulmonary hemorrhage, high cryocrit levels and type II mixed cryoglobulinemia are also associated with severe prognosis [20].

It has been demonstrated that most manifestations of mixed cryoglobulinemia have a strong correlation with the $\mathrm{HCV}$ virological response and respond to clearance of $\mathrm{HCV}$ during antiviral therapy [21]. In fact, patients show a good symptomatic response to antiviral treatment, even in the absence of a sustained virological response [22]. Therefore, patients with mild to moderate disease should be given optimal antiviral therapy. Pegylated interferon (IFN) and ribavirin are effective for this purpose. However, a cautious attitude could be suggested in consideration of the side effects possibly induced by IFN in the context of mixed cryoglobulinemia (for example, possible worsening of peripheral neuropathy or skin ulcers). Besides, direct-acting antiviral (DAA) agents appear to be effective in the treatment of mixed cryoglobulinemia, with an excellent safety profile. The association of pegylated 
IFN, ribavirin and boceprevir or telaprevir is associated with improved rated of sustained virological response and remission of cryoglobulinemia in genotype 1 [23]. Sofosbuvir appears to be associated with high rates of sustained virological response in patients with mixed cryoglobulinemia, improving not only liver disease, but also extrahepatic manifestations such as arthralgia, purpura or peripheral neuropathy, with high rates of clinical response of vasculitis $[24,25]$. However, even with successful clearance of the virus, the symptomatic response may lag, particularly in those with severe or life-threatening vasculitis [26,27]. For this reason, patients with severe mixed cryoglobulinemia (i.e., worsening of renal function, mononeuritis multiplex, extensive skin disease, intestinal ischemia, etc.), require control of the disease with rituximab, with or without plasmapheresis, before the initiation of optimal antiviral therapy [28]. Other immunosuppressant drugs, such as cyclophosphamide, may also be used in more severe or refractory cases [29].

\section{HCV-associated arthritis}

Polyarthralgia is the most common rheumatologic symptom associated with hepatitis $\mathrm{C}$, being reported in 40$80 \%$ of patients [5]. HCV-related arthritis may be divided into arthritis associated with mixed cryoglobulinemia and arthritis not associated with mixed cryoglobulinemia [30]. Articular involvement is usually bilateral and symmetrical, and preferentially involves small articulations, such as the metacarpophalangeal, proximal interphalangeal and wrist articulations, mimicking rheumatoid arthritis (RA). Knees may also be involved. Rheumatoid factor (RF) is positive in up to $80 \%$ of patients with mixed cryoglobulinemia, although its presence is not correlated with articular disease [15]. The absence of articular erosion and deformity, subcutaneous nodules and anti-C citrullinated peptides (anti-CCP), more specific for RA, may be helpful in distinguishing RF from RA (Table 2).

A distinction between the symptoms related to chronic HCV infection or to a newly developed rheumatologic disorder is fundamental for therapeutic decisions. In fact, the therapeutic approach to HCV-related arthritis remains largely empirical. Mainstream treatment based on the administration of hydroxychloroquine plus low doses of glucocorticoids and/or non-steroidal anti-inflammatory drugs is still largely preferred [31,32]. Cyclosporine represents a useful alternative because of its antiviral properties [33]. Anti-tumor necrosis factor agents are safe, but may be considered excessive for such mild disorders [34]. The effect of DAA in HCV-associated arthritis is currently unknown.

\section{Sicca syndrome/Sjögren's syndrome (SS)}

Sicca symptoms of either the mouth or eyes have been reported in $20-30 \%$ of patients with HCV infection, whereas less than $5 \%$ of patients with SS are HCV-positive [5]. There
Table 2 Distinction between rheumatoid arthritis and $\mathrm{HCV}$-associated arthritis

\begin{tabular}{lll}
\hline Characteristic & $\begin{array}{l}\text { Rheumatoid } \\
\text { arthritis }\end{array}$ & $\begin{array}{l}\text { HCV-associated } \\
\text { arthritis }\end{array}$ \\
\hline Rheumatoid factor & Up to $85 \%$ & Up to 70\% \\
\hline Anti-CCP & $55-99 \%$ & $2-4 \%$ \\
\hline ANA & $30 \%$ & $10 \%$ \\
\hline Cryoglobulins & $1 \%$ & $40-55 \%$ \\
\hline Anti-thyroid antibodies & Up to 30\% & Up to $10 \%$ \\
\hline Erosive arthritis & Present & Absent \\
\hline Subcutaneous nodules & May be present & Absent \\
\hline Risk factors for HCV & Unrelated & May be present \\
\hline
\end{tabular}

HCV, hepatitis $C$ virus; anti-CCP, anti-C citrullinated peptides; ANA, antinuclear antibodies

are many clinical and histological similarities between HCVrelated Sicca syndrome and primary SS. However, a typical SS, defined by the presence of xerostomia, xerophthalmia, antiSSA or anti-SSB antibodies and typical salivary gland histology, is rarely found in patients with $\mathrm{HCV}$ infection. Besides, patients with HCV-positive SS are usually older and more likely to have photosensitivity, mixed cryoglobulinemia and arthralgia than patients with primary SS. Low titers of antinuclear antibodies (ANA) and RF are common in patients with HCV-related Sicca syndrome, but the presence of anti-SSA or anti-SSB autoantibodies is rare [9].

In a multicenter study involving 137 patients with both SS and HCV infections, 58\% presented a systemic process showing diverse extra-glandular manifestations, with articular involvement (44\%), vasculitis (20\%), and neuropathy (16\%) being the most frequent features observed. The main immunologic features were ANA (65\%), hypocomplementemia (51\%), and cryoglobulinemia (50\%). Only 23\% patients had positive anti-Ro/SS-A and/or anti-La/SS-B antibodies, and these had a higher prevalence of some extra-glandular features [35].

Treatment of HCV infection with IFN alone results in severe immunological complications in more than half of the patients. Ribavirin combined with IFN gives a significative sustained virological response and could lower the incidence of immunologic interferon-mediated complications, with a favorable outcome of Sicca syndrome [36].

\section{Autoimmunity/antibodies}

At least one immunological abnormality is present in up to $50 \%$ of $\mathrm{HCV}$-infected patients [15]. Autoantibodies are frequently present in chronic hepatitis $\mathrm{C}$, the most common being RF and cryoglobulins, already mentioned (Table 3). Although the pathophysiology of these autoimmune processes is not entirely understood, it is thought to be related to HCV's influence on immune system cells, particularly B lymphocytes. Nevertheless, such autoantibodies have not been associated 
Table 3 Frequencof autoantibodies in chronic hepatitis C

\begin{tabular}{lc} 
Type of autoantibody & Frequency (\%) \\
\hline Rheumatoid factor & $45-70$ \\
Cryoglobulins & $40-60$ \\
\hline Antinuclear antibodies & $10-40$ \\
Anti-smooth muscle & $7-27$ \\
Anti-cardiolipin & $15-30$ \\
Anti-thyroid & $5-12$ \\
Anti-mitochondrial & 1 \\
\hline
\end{tabular}

with connective tissue disease manifestations, except for mixed cryoglobulins [37].

There are expectations that a better knowledge of these autoimmune processes may help understand the pathophysiology of rheumatologic manifestations of hepatitis C, which has obvious clinical, prognostic and therapeutic implications. Accordingly, over the recent years, various antibodies and their relationships with chronic hepatitis $\mathrm{C}$ rheumatologic manifestations have been studied intensively.

$\mathrm{Clq}$ is the first component of the classical pathway of complement activation, playing an important role in the degradation of immune complexes, with the inactivation of this process possibly playing a role in immunity. Interestingly, a study demonstrated a higher prevalence of anti-C1q autoantibodies among patients with HCV and rheumatologic manifestations, compared to patients with $\mathrm{HCV}$ without these manifestations. This study also demonstrated an increased prevalence of anti-C1q autoantibodies in patients infected with HCV with mixed cryoglobulinemia and lymphoma, opening the possibility of their use as a biomarker for the diagnosis of lymphoma [38].

Osteopontin plays a role in inflammation, autoimmunity and fibrosis, and its role in chronic hepatitis $\mathrm{C}$ has been investigated. Interestingly, higher levels of osteopontin were seen in patients with HCV and rheumatologic manifestations, and it could thus be used as marker for rheumatologic manifestations associated with $\mathrm{HCV}$, particularly vasculitis and cryoglobulinemia [39].

\section{Other diseases and their association with HCV}

Systemic lupus erythematosus (SLE) may share clinical and serological characteristics with rheumatologic manifestations of chronic hepatitis C, such as arthralgia, myalgia, Sicca syndrome, and ANA. In fact, some studies demonstrate a higher prevalence of $\mathrm{HCV}$ infection among patients with SLE than in the general population [40] and, although the association between HCV and SLE is not clear, it is recommended to screen patients with SLE for HCV.

Polyarteritis nodosa, though more commonly associated with hepatitis B, may also occur in association with hepatitis $\mathrm{C}$, with an estimated risk of up to $12 \%$ in chronic
HCV infection [41]. HCV-associated polyarteritis nodosa is characterized by more severe symptoms, although with higher clinical remission rates [42]. Antiviral therapies and corticosteroids are the cornerstone treatments for HCV-related polyarteritis nodosa. Plasma exchange, cyclophosphamide and rituximab may be tried in resistant cases [43].

$\mathrm{HCV}$-infected patients may also present with diffuse bone pain secondary to osteosclerosis. Its pathogenesis is not fully understood, but is probably related to dysregulation of osteoprotegerin/RANKL, with predominance of osteoprotegerin [44].

On the other hand, despite the fact that anti-cardiolipin antibodies are present in up to $30 \%$ of $\mathrm{HCV}$-infected patients, an increase in thrombotic complications has not been demonstrated and an association between antiphospholipid syndrome and HCV infection has yet to be proven [45]. Although up to $20 \%$ of $\mathrm{HCV}$-infected patients present with fibromyalgia, this association also has yet to be proven [46].

Despite the effectiveness demonstrated in patients with mixed cryoglobulinemia, the impact of new DAAs on other rheumatologic manifestations is not clear [47]. For fibromyalgia, Younossi et al recently reported major benefits of sofosbuvir-based DAAs on most patient-reported outcomes, including mental and physical fatigue, at week 12 and week 24 post-treatment. A beneficial effect of DAAs was also suggested on the cerebral magnetic resonance signal in the basal ganglia, correlated with the virological response [48].

\section{Hepatitis B}

\section{General considerations}

Hepatitis B is another disease with high world prevalence rates that affects primarily the liver and may also cause extrahepatic manifestations that, though less common than in hepatitis C, may be found in about $20 \%$ of patients infected with either acute or chronic hepatitis [49] The pathogenesis of the extrahepatic manifestations associated with hepatitis B virus (HBV) is not entirely understood, but it is thought that they are mediated by HBs and HBe immunocomplexes [50] or viral replication in extrahepatic tissues [51]. No correlation has been found between the type of extrahepatic manifestation and the HBV genotype [52].

\section{Polyarteritis nodosa}

Polyarteritis nodosa is a small- and medium-sized vessel systemic vasculitis that affects typically visceral and renal vessels, sparing the pulmonary circulation. It is mediated by immunocomplex deposition and clinically characterized by abdominal pain, hypertension, rash, polyarthritis, and weight loss.

The association between polyarteritis nodosa and hepatitis $\mathrm{B}$ is well established. Although polyarteritis nodosa $\mathrm{s}$ a rare complication of chronic hepatitis B, occurring in less than 5\% of those infected [53], HBs is positive in about $50 \%$ of patients 
with polyarteritis nodosa [54]. The activity of the disease is proportional to the level of circulating immunocomplexes [55].

HBV-associated polyarteritis nodosa usually manifests in the first 6 months of infection and, in comparison with its classic form, is associated with a higher frequency of gastrointestinal complications, orchitis, severe hypertension and renal infarct [54], and a lower frequency of antineutrophilic cytoplasmic antibody positivity [56].

The immunosuppressant treatment used in the classic form of the disease is not indicated in HBV-associated polyarteritis nodosa. Currently, the most accepted treatment strategies involve a combination of plasmapheresis, glucocorticoids, and antiviral therapy, since suppressing viral replication usually results in disease resolution [57]. An excellent response to a combination of glucocorticoids and entecavir has been demonstrated. Similar results are expected for tenofovir [58].

\section{HBV-associated arthritis}

Polyarthritis and polyarthralgia may be seen during the prodromal period of acute viral hepatitis of any etiology, and especially in patients with HBV. In up to $30 \%$ of cases of acute hepatitis $\mathrm{B}$, there is a prodromal period, and joint symptoms frequently precede jaundice. Patients may complain of symmetrical, non-destructive polyarthritis, mostly involving small articulations in hands and feet and associated with skin manifestations [57].

These symptoms usually subside as the typical features of hepatitis appear, leaving no residual deformities, and usually no treatment is necessary [59]. However, an asymmetrical nondestructive polyarthritis associated with erythematous skin lesions may persist, even in chronic hepatitis B [56].

\section{Hepatitis A}

Although extrahepatic manifestations are less common in patients with hepatitis A virus (HAV) than in those with hepatitis $\mathrm{B}$ or $\mathrm{C}$, occasionally, patients with $\mathrm{HAV}$ infection manifest symptoms related to vasculitis, arthritis, and cryoglobulinemia, consistent with the formation of circulating immune complexes $[60,61]$. In fact, acute hepatitis A may be associated with evanescent rash in approximately $14 \%$ of cases and with arthralgias in approximately $11 \%$. Both these manifestations usually occur in an early phase and are usually transient, with complete resolution [62].

Hepatitis A may also be associated with adultonset Still's disease, a systemic inflammatory disease characterized by high fever accompanied by systemic manifestations, such as arthralgia, rash, lymphadenopathy and hepatosplenomegaly $[63,64]$.

\section{Hepatitis E}

Hepatitis E virus (HEV) is an important public health problem in the developing world; it afflicts almost 20 million individuals annually and causes acute liver injury in 3.5 million. It is implicated in approximately 56,000 deaths, with the highest prevalence in East and South Asia. Nevertheless, HEV infection is becoming an emergent disease in developed countries. It was traditionally thought to occur in individuals traveling to areas where it is endemic; however, sporadic autochthonous hepatitis $\mathrm{E}$ has been increasingly diagnosed among individuals with no history of recent travel [65].

As with other viral hepatitis, numerous extrahepatic manifestations are reported in association with acute or chronic HEV infection. Acute pancreatitis and neurological, musculoskeletal, hematological, renal and other immunemediated manifestations have been described. These extrahepatic manifestations can overshadow the hepatic injury and HEV may not be suspected [65].

Rheumatologic manifestations have also been reported in association with hepatitis E. In fact, acute HEV infection may present as an acute polyarthritis, with symmetric involvement of ankles, knees, hips, hands, wrists, elbows and/or shoulders, with or without swelling, usually self-limited and resolving spontaneously with HEV clearance [66-68]. Accordingly, HEV infection must be considered in patients presenting with a background of polyarthralgia and elevated liver enzymes.

The pathogenesis of $\mathrm{HEV}$-associated rheumatologic manifestations is not completely understood. However, $\mathrm{HEV}$ infection is known to be associated with mixed cryoglobulinemia [69], which may develop during the course of chronic hepatitis E and resolve with HEV clearance [70], or may even occur after HEV clearance, with good response to immunosuppressive treatment [71].

\section{Autoimmune hepatitis (AIH)}

Rheumatologic manifestations are common in AIH. In fact, hypergammaglobulinemia and autoantibodies are common, and the most common extrahepatic manifestations are arthralgia and skin rash [72,73]. AIH is separate from rheumatologic disorders, but the boundaries between them can be indistinct. In fact, over $30 \%$ of patients with $\mathrm{AIH}$ have concurrent immune-mediated manifestations, and the rheumatologic features in these patients may mask the underlying liver disease. In contrast, $25 \%$ of patients with primary rheumatologic diseases have coincidental liver dysfunction, and they may be misclassified and treated as primary liver disease [73].

Certain clinical features may be useful to establish a distinction between coexisting distinct autoimmune diseases and AIH-related rheumatologic manifestations. Elevation of liver enzymes is more common in $\mathrm{AIH}$ and the presence of certain autoantibodies is more specific to AIH (for example, anti-smooth muscle in type $1 \mathrm{AIH}$ or anti-LKM1 and anti-LC1 in type $2 \mathrm{AIH}$ ). On the other hand, some clinical manifestations, like Sicca symptoms in SS or Raynaud or inflammatory arthralgia in RA, may help in the differential diagnosis [74].

Elderly patients with AIH have a higher frequency of rheumatologic conditions than young adults, including RA, 
SS, and SLE [75], underscoring the importance of pursuing the diagnosis of $\mathrm{AIH}$ in the elderly with liver dysfunction and searching beyond the rheumatologic manifestations. The importance of excluding AIH in patients with rheumatologic features is further reinforced by studies in primary SS that demonstrated the presence of background autoimmune liver disease in $47 \%$ of patients [76] and the association between systemic sclerosis and polymyositis and $\mathrm{AIH}$ [77].

Anti-CCP are found in $9 \%$ of patients with $\mathrm{AIH}$, demonstrating a tight association with erosive arthritis, thereby identifying patients with liver disease, more prone to develop inflammatory joint disease [78]. Patients with AIH and antiCCP also have a greater occurrence of histological cirrhosis and hepatic failure, making these autoantibodies possible surrogate markers of advanced hepatic fibrosis in AIH [79].

Standard treatment for AIH is based on corticosteroids, as monotherapy or in association with azathioprine. An accurate distinction between AIH and other primary autoimmune diseases is essential when considering a therapeutic strategy. On the one hand, some drugs used in the treatment of primary rheumatologic diseases, like methotrexate, are potentially hepatotoxic. On the other, though azathioprine is effective in the treatment of AIH-related rheumatologic manifestations, its effectiveness is limited in primary diseases, including RA [80].

\section{Alcoholic liver disease (ALD)}

ALD is often accompanied by a wide variety of extrahepatic manifestations. In general, progressive tissue damage may be found in virtually every organ and there is a positive correlation between extrahepatic manifestations and the degree of liver damage [81].

ALD is associated with decreased bone mineral density [82-85]. Hepatic osteodystrophy generically defines the overall alterations in bone mineral density (osteoporosis or osteopenia) secondary to chronic liver disease [82]. The prevalence of osteopenia in ALD patients ranges between 34\% and $48 \%$, and the prevalence of osteoporosis ranges between $11 \%$ and $36 \%$ [83]. Ethanol may be responsible for both decreased bone synthesis and increased bone breakdown. In addition, systemic alterations, such as malnutrition, malabsorption, liver disease, increased levels of proinflammatory cytokines, alcoholic myopathy and neuropathy, low testosterone levels and an increased risk of trauma, play contributory roles [84].

Treatment includes smoking and alcohol cessation and general measures such as changes in nutrition and exercise. Calcium and vitamin D supplements are recommended in all patients with ALD and osteoporosis. Bisphosphonates have been proven to be effective in increasing bone mineral density in chronic liver disease, and they can be used in the setting of ALD. Alternatives include raloxifene, hormone replacement therapy and calcitonin $[83,85]$. Interestingly, a recent metaanalysis demonstrated a significant association between ALD and an increased risk of bone fractures, but not between ALD and osteoporosis [86].

\section{Nonalcoholic fatty liver disease (NAFLD)}

NAFLD is reported to be associated with extrahepatic diseases, including cardiovascular disease, diabetes mellitus, and thyroid gland abnormalities. Although the underlying pathogenesis is not clear, there is also an association between NAFLD and low bone mineral density in the forms of osteoporosis and osteopenia [87]. In fact, in a cross-sectional study involving 231 subjects, the presence of significant liver fibrosis assessed via transient elastography was independently associated with low bone mineral density, osteopenia and osteoporosis in NAFLD subjects [88]. Interestingly, there could be potential sex-specific differences in the effect of NAFLD on bone mineral density, as another study reported a negative effect of NAFLD on bone mineral density in men, despite a positive effect on lumbar spine bone mineral density in postmenopausal women [89]. Screening and surveillance of the skeletal system for osteoporosis in patients with NAFLD may be considered in future strategies and guidelines for NAFLD management.

Pentoxifylline has demonstrated promising results, with improvement in liver enzyme abnormalities and insulin resistance [90] that could also be beneficial to other systemic manifestations of the disease, including bone mineral density. Some other drugs in phase III trials (for example obeticholic acid and elafibranor) demonstrated improvements in liver fibrosis [91].

\section{Primary biliary cholangitis (PBC)}

$\mathrm{PBC}$ is a chronic cholestatic hepatic disease, where $95 \%$ of patients present with antimitochondrial antibodies (AMA), demonstrating a prominent autoimmune basis. Approximately $30-50 \%$ of patients present with osteoporosis, osteopenia or osteomalacia secondary to cholestasis, and vitamin D deficiency [92].

In addition, up to $60 \%$ of patients may present with an extrahepatic autoimmune manifestation [93]. Among the rheumatologic manifestations, systemic sclerosis is the most common. Studies suggest hepatobiliary involvement in approximately $7.5 \%$ of patients with systemic sclerosis, PBC being the most common disorder [94] CREST variant (calcinosis, Raynaud syndrome, esophageal dysmotility, sclerodactyly, and teleangiectasia) has been reported in up to $15 \%$ of Italian patients with PBC [93]. The presence of these manifestations and/or serological abnormalities should alert the gastroenterologist to a need for early referral to rheumatology.

Up to $50 \%$ of patients may present with symptomatic inflammatory arthropathy. Arthritis is a frequent complaint in otherwise asymptomatic patients and may be observed in the preclinical phase. The characteristics of the arthritis are highly variable, and $\mathrm{PBC}$ may be associated with distinct types of arthropathy, including RA, SLE, and ankylosing spondylitis [93].

Even though ANA positivity (50-70\%) is not particularly helpful in the differential diagnosis versus other autoimmune 
diseases, certain features like AMA positivity (in almost 100\% of cases) or cholestatic pattern in liver function tests are important. This distinction is important, since the mainstay of treatment for PBC is ursodesoxycholic acid, quite different from other autoimmune diseases [74].

\section{Hemochromatosis}

Hemochromatosis is an autosomal recessive disorder of iron metabolism in which the body accumulates excessive iron. The deposition of excessive iron in a variety of organs leads to their failure, with manifestations that include cirrhosis, diabetes mellitus, cardiomyopathy, arthritis and hypogonadism.

Arthritis appears to be the most common extrahepatic clinical feature of hemochromatosis, affecting more than half of patients. Arthritic symptoms may present before other visceral manifestations become evident and in some cases arthritis may be the sole clinical manifestation. Hemochromatosis arthritis is typically a polyarthropathy, involving the metacarpophalangeal, proximal interphalangeal, wrist, knee and vertebral joints, and less commonly the hip. Although the arthritis is typically symmetric, it may be unilateral [2]. Arthritis is progressive in $75 \%$ of cases, stable in $12.5 \%$ and improving in $12.5 \%$ and, although iron removal by chelation or phlebotomy may favorably affect the overall course of hemochromatosis, there is usually no improvement in articular symptoms [95]. Arthropathy may actually improve, remain stable or worsen with treatment of hemochromatosis. Treatment is similar to that of idiopathic osteoarthrosis and is based on analgesia [96].

Chondrocalcinosis may occur in up to $50 \%$ of patients, involving mainly knee and wrist joints. Colchicine may be used to prevent attacks of pseudogout. Given the importance of interleukin (IL)-1 in arthropathies mediated by deposition of crystals [97], anakinra (inhibitor of IL-1) has been reported to be effective in the treatment of severe hemochromatosisassociated arthropathy involving hands, with symptomatic improvement and resolution of inflammatory signs [98].

\section{Wilson's disease}

Wilson's disease is a disease of copper metabolism, resulting in excessive accumulation of this metal. Skeletal involvement is common. Patients may complain of pain and stiffness, mainly in the knees, wrists, or other large joints, with synovial copper accumulation. Other rheumatologic complications include osteomalacia, osteoporosis, and premature osteoarthrosis [2].

\section{Concluding remarks}

Rheumatologic manifestations of hepatic diseases may complicate the diagnostic approach and alter the natural history of the primary disease, sometimes worsening the prognosis as a result of the associated multiple organ dysfunction. The prevalence of these manifestations can be variable according to different diseases. It is necessary not only for rheumatologists, but also for other clinicians, to be aware that these atypical manifestations may reflect an undiagnosed hepatic disease. Despite the advances achieved so far, further studies are necessary to provide a better understanding of the pathogenesis of the rheumatologic manifestations of hepatic diseases in order to identify new biomarkers for these manifestations and new therapeutic targets. On the other hand, in a patient with known hepatic disease who presents with rheumatologic symptoms, it is crucial to make an accurate distinction between a rheumatologic manifestation of hepatic disease and primary rheumatologic disease, since the treatment is often different.

Whenever there are extrahepatic manifestations, including arthralgia, xerostomia, xerophthalmia, rash, among others, or serological abnormalities, it may be wise to refer the patient to a rheumatologist. In addition, gastroenterologists must keep in mind the possible osteoarticular symptoms of therapies employed in the treatment of gastrointestinal diseases. We hope this review can inspire the creation of protocols with characteristics of rheumatologic manifestations that should alert the physician to the possibility of their being secondary to hepatic diseases, so that these can be screened ad initium, avoiding late diagnosis and thereby improving prognosis. It is also important that, in these cases, there is a multidisciplinary approach, with a close collaboration between gastroenterologists and rheumatologists, in order to provide the best treatment for the patient.

\section{References}

1. Ramos-Remus C, Bahlas S, Vaca-Morales O. Rheumatic features of gastrointestinal tract, hepatic, and pancreatic diseases. Curr Opin Rheumatol 1997;9:56-61.

2. Chi ZC, Ma SZ. Rheumatologic manifestations of hepatic diseases. Hepatobiliary Pancreat Dis Int 2003;2:32-37.

3. Zignego AL, Gragnani L, Giannini C, Laffi G. The hepatitis C virus infection as a systemic disease. Intern Emerg Med 2012; 7(Suppl 3):S201-S208.

4. Negro F. Epidemiology of hepatitis C in Europe. Dig Liv Dis 2014;46(Suppl 5):S158-S164.

5. Cacoub P, Renou C, Rosenthal E, et al. Extrahepatic manifestations associated with hepatitis $\mathrm{C}$ virus infection. A prospective multicenter study of 321 patients. The GERMIVIC. Groupe d'Etude et de Recherche en Medecine Interne et Maladies Infectieuses sur le Virus de l'Hepatite C. Medicine (Baltimore) 2000;79:47-56.

6. Zignego AL, Giannini C, Monti M, Gragnani L. Hepatitis C virus lymphotropism: lessons from a decade of studies. Dig Liv Dis 2007;39(Suppl 1):S38-S45.

7. Polo ML, Laufer N. Extrahepatic manifestations of HCV: the role of direct acting antivirals. Expert Rev Anti Infect Ther 2017; 15:737-746.

8. Zignego AL, Ferri C, Giannini C, et al. Hepatitis C virus infection in mixed cryoglobulinemia and B-cell non-Hodgkin's lymphoma: evidence for a pathogenetic role. Arch Virol 1997;142:545-555.

9. Cacoub P, Comarmond C, Domont F, Savey L, Desbois AC, Saadoun D. Extrahepatic manifestations of chronic hepatitis C virus infection. Ther Adv Infect Dis 2016;3:3-14. 
10. Lormeau C, Falgarone G, Roulot D, Boissier MC. Rheumatologic manifestations of chronic hepatitis C infection. Joint Bone Spine 2006;73:633-638.

11. Sansonno D, Cornacchiulo V, Iacobelli AR, Di Stefano R, Lospalluti M, Dammacco F. Localization of hepatitis C virus antigens in liver and skin tissues of chronic hepatitis $\mathrm{C}$ virusinfected patients with mixed cryoglobulinemia. Hepatology 1995;21:305-312.

12. Ferri C, Giuggioli D, Cazzato M, Sebastiani M, Mascia MT, Zignego AL. HCV-related cryoglobulinemic vasculitis: an update on its etiopathogenesis and therapeutic strategies. Clin Experiment Rheumatol 2003;21(6 Suppl 32):S78-S84.

13. Viganò M, Lampertico P, Rumi MG, et al. Natural history and clinical impact of cryoglobulins in chronic hepatitis $\mathrm{C}$ : 10-year prospective study of 343 patients. Gastroenterology 2007;133:835-842.

14. Zignego AL, Ferri C, Pileri SA, Caini P, Bianchi FB; Italian Association of the Study of Liver Commission on Extrahepatic Manifestations of HCV infection. Extrahepatic manifestations of hepatitis $C$ virus infection: a general overview and guidelines for a clinical approach. Dig Liver Dis 2007;39:2-17.

15. Cacoub P, Gragnani L, Comarmond C, Zignego AL. Extrahepatic manifestations of chronic hepatitis C virus infection. Dig Liver Dis 2014;46(Suppl 5):S165-S173.

16. De Vita S, Quartuccio L, Salvin S, Corazza L, Zabotti A, Fabris M. Cryoglobulinaemia related to Sjogren's syndrome or HCV infection: differences based on the pattern of bone marrow involvement, lymphoma evolution and laboratory tests after parotidectomy. Rheumatology (Oxford) 2012;51:627-633.

17. Quartuccio L, Isola $\mathrm{M}$, Corazza L, et al. Performance of the preliminary classification criteria for cryoglobulinaemic vasculitis and clinical manifestations in hepatitis $\mathrm{C}$ virusunrelated cryoglobulinaemic vasculitis. Clin Exp Rheumatol 2012;30(1 Suppl 70):S48-S52.

18. Retamozo S, Diaz-Lagares C, Bosch X, et al. Life-threatening cryoglobulinemic patients with hepatitis $\mathrm{C}$ : clinical description and outcome of 279 patients. Medicine (Baltimore) 2013;92:273-284.

19. Cacoub P, Comarmond C. New insights into HCV-related rheumatologic disorders: a review. J Adv Res 2017;8:89-97.

20. Ramos-Casals M, Robles A, Brito-Zerón P, et al. Life-threatening cryoglobulinemia: clinical and immunological characterization of 29 cases. Semin Arthritis Rheum 2006;36:189-196.

21. Cacoub P, Saadoun D, Limal N, Sene D, Lidove O, Piette JC. PEGylated interferon alfa-2b and ribavirin treatment in patients with hepatitis C virus-related systemic vasculitis. Arthritis Rheum 2005;52:911-915.

22. Joshi S, Kuczynski M, Heathcote EJ. Symptomatic and virological response to antiviral therapy in hepatitis $\mathrm{C}$ associated with extrahepatic complications of cryoglobulimia. Dig Dis Sci 2007;52:2410-2417.

23. Gragnani L, Fabbrizzi A, Triboli E, et al. Triple antiviral therapy in hepatitis C virus infection with or without mixed cryoglobulinaemia: a prospective, controlled pilot study. Dig Liver Dis 2014;46:833-837.

24. Mazzaro C, Dal Maso L, Quartuccio L, et al. Long-term effects of the new direct antiviral agents (DAAs) therapy for HCV-related mixed cryoglobulinaemia: a multicentre open-label study. Clin Experiment Rheumatol 2018;36(Suppl 111):107-114.

25. Gragnani L, Visentini M, Fognani E, et al. Prospective study of guideline-tailored therapy with direct-acting antivirals for hepatitis C virus-associated mixed cryoglobulinemia. Hepatology 2016;64:1473-1482.

26. Emery JS, Kuczynski M, La D, et al. Efficacy and safety of direct acting antivirals for the treatment of mixed cryoglobulinemia. Am J Gastroenterol 2017;112:1298-1308.

27. Martin P, Fabrizi F. Editorial: Benefit of direct-acting antiviral therapy for cryoglobulinemia due to hepatitis $\mathrm{C}$ infection. Am J
Gastroenterol 2017;112:1309-1310.

28. Saadoun D, Delluc A, Piette JC, Cacoub P. Treatment of hepatitis C-associated mixed cryoglobulinemia vasculitis. Curr Opin Rheumatol 2008;20:23-28.

29. Saadoun D, Pineton de Chambrun M, Hermine O, et al. Using rituximab plus fludarabine and cyclophosphamide as a treatment for refractory mixed cryoglobulinemia associated with lymphoma. Arthritis Care Res (Hoboken) 2013;65:643-647.

30. Rosner I, Rozenbaum M, Toubi E, Kessel A, Naschitz JE, Zuckerman E. The case for hepatitis C arthritis. Semin Arthritis Rheum 2004;33:375-387.

31. Zuckerman E, Yeshurun D, Rosner I. Management of hepatitis C virus-related arthritis. BioDrugs 2001;15:573-584.

32. Palazzi C, D’Amico E, D'Angelo S, Gilio M, Leccese P, Olivieri I. An update on the management of hepatitis $C$ virus-related arthritis. Expert Opin Pharmacother 2014;15:2039-2045.

33. Galeazzi M, Bellisai F, Manganelli S, Morozzi G, Sebastiani GD. Cyclosporine A for the treatment of autoimmune disorders in HCV infected patients. Autoimmun Rev 2006;5:493-498.

34. Marotte H, Fontanges E, Bailly F, Zoulim F, Trepo C, Miossec P. Etanercept treatment for three months is safe in patients with rheumatological manifestations associated with hepatitis $\mathrm{C}$ virus. Rheumatology (Oxford) 2007;46:97-99.

35. Ramos-Casals M, Loustaud-Ratti V, De Vita S, et al; SS-HCV Study Group. Sjögren syndrome associated with hepatitis C virus: a multicenter analysis of 137 cases. Medicine (Baltimore) 2005;84:81-89.

36. Doffoël-Hantz V, Loustaud-Ratti V, Ramos-Casals M, et al. [Evolution of Sjögren syndrome associated with hepatitis $\mathrm{C}$ virus when chronic hepatitis $\mathrm{C}$ is treated by interferon or the association of interferon and ribavirin]. Rev Med Interne 2005;26:88-94.

37. Vassilopoulos D, Calabrese LH. Extrahepatic immunological complications of hepatitis C virus infection. AIDS 2005; 19(Suppl 3):S123-S127.

38. Fadda SH, Bassyouni IH, Hamdy A, Foad NA, Wali IE. Anti-C1q in chronic hepatitis $\mathrm{C}$ virus genotype IV infection: association with autoimmune rheumatologic manifestations. Immunol Invest 2015;44:45-55.

39. Bassyouni IH, Bassyouni RH, Ibrahim NH, Soliman AF. Elevated serum osteopontin levels in chronic hepatitis $\mathrm{C}$ virus infection: association with autoimmune rheumatologic manifestations. J Clin Immunol 2012;32:1262-1269.

40. Ahmed MM, Berney SM, Wolf RE, et al. Prevalence of active hepatitis C virus infection in patients with systemic lupus erythematosus. Am J Med Sci 2006;331:252-256.

41. Pagnoux C, Seror R, Henegar C, et al; French Vasculitis Study Group. Clinical features and outcomes in 348 patients with polyarteritis nodosa: a systematic retrospective study of patients diagnosed between 1963 and 2005 and entered into the French Vasculitis Study Group Database. Arthritis Rheum 2010;62:616-626.

42. Saadoun D, Terrier B, Semoun O, et al. Hepatitis C virus-associated polyarteritis nodosa. Arthritis Care Res (Hoboken) 2011;63:427-435.

43. de Menthon M, Mahr A. Treating polyarteritis nodosa: current state of the art. Clin Exp Rheumatol 2011;29(1 Suppl 64):S110-S116.

44. Fiore CE, Riccobene S, Mangiafico R, Santoro F, Pennisi P. Hepatitis C-associated osteosclerosis $(\mathrm{HCAO})$ : report of a new case with involvement of the OPG/RANKL system. Osteoporos Int 2005; 16:2180-2184.

45. Mangia A, Margaglione M, Cascavilla I, et al. Anticardiolipin antibodies in patients with liver disease. Am J Gastroenterol 1999;94:2983-2987.

46. Kozanoglu E, Canataroglu A, Abayli B, Colakoglu S, Goncu K. Fibromyalgia syndrome in patients with hepatitis $\mathrm{C}$ infection. Rheumatol Int 2003;23:248-251.

47. Cacoub P, Commarmond C, Sadoun D, Desbois AC. Hepatitis C virus infection and rheumatic diseases: the impact of direct-acting 
antiviral agents. Rheum Dis Clin North Am 2017;43:123-132.

48. Younossi ZM, Stepanova M, Mishra A, Venkatesan C, Henry L, Hunt $S$. The impact of chronic hepatitis $C$ on resource utilisation and in-patient mortality for Medicare beneficiaries between 2005 and 2010. Aliment Pharmacol Ther 2013;38:1065-1075.

49. Cacoub P, Terrier B. Hepatitis B-related autoimmune manifestations. Rheum Dis Clin North Am 2009;35:125-137.

50. Mason A. Role of viral replication in extrahepatic syndromes related to hepatitis B virus infection. Minerva Gastroenterol Dietol 2006;52:53-66.

51. Mason A, Theal J, Bain V, Adams E, Perrillo R. Hepatitis B virus replication in damaged endothelial tissues of patients with extrahepatic disease. Am J Gastroenterol 2005;100:972-976.

52. Cacoub $P$, Saadoun D, Bourlière $M$, et al. Hepatitis $B$ virus genotypes and extrahepatic manifestations. J Hepatol 2005;43:764-770.

53. Guillevin L, Mahr A, Callard P, et al; French Vasculitis Study Group. Hepatitis B virus-associated polyarteritis nodosa: clinical characteristics, outcome, and impact of treatment in 115 patients. Medicine (Baltimore) 2005;84:313-322.

54. Guillevin L, Lhote F, Cohen P, et al. Polyarteritis nodosa related to hepatitis B virus. A prospective study with long-term observation of 41 patients. Medicine (Baltimore) 1995;74:238-253.

55. Fye KH, Becker MJ, Theofilopoulos AN, Moutsopoulos H, Feldman JL, Talal N. Immune complexes in hepatitis B antigenassociated periarteritis nodosa. Detection by antibody-dependent cell-mediated cytotoxicity and the Raji cell assay. Am J Med 1977;62:783-791.

56. Han SH. Extrahepatic manifestations of chronic hepatitis B. Clin Liver Dis 2004;8:403-418.

57. Satsangi S, Gupta N. Rheumatologic manifestations of viral hepatitis B and C. J Hepat 2015;1:1-5.

58. Naniwa T, Maeda T, Shimizu S, Ito R. Hepatitis B virus-related polyarteritis nodosa presenting with multiple lung nodules and cavitary lesions. Chest 2010;138:195-197.

59. Trepo C, Guillevin L. Polyarteritis nodosa and extrahepatic manifestations of HBV infection: the case against autoimmune intervention in pathogenesis. J Autoimmun 2001;16:269-274.

60. Inman RD, Hodge M, Johnston ME, Wright J, Heathcote J. Arthritis, vasculitis, and cryoglobulinemia associated with relapsing hepatitis A virus infection. Ann Intern Med 1986;105:700-703.

61. Ilan Y, Hillman M, Oren R, Zlotogorski A, Shouval D. Vasculitis and cryoglobulinemia associated with persisting cholestatic hepatitis A virus infection. Am J Gastroenterol 1990;85:586-587.

62. Schiff ER. Atypical clinical manifestations of hepatitis A. Vaccine 1992;10(Suppl 1):S18-S20.

63. Seo SR, Kim SS, Lee SJ, Kim TJ, Park YW, Lee SS. Adult-onset Still disease in a patient with acute hepatitis A. J Clin Rheumatol 2011;17:444-445.

64. Sridharan S, Mossad S, Hoffman G. Hepatitis A infection mimicking adult onset Still's disease. J Rheumatol 2000;27:1792-1795.

65. Bazerbachi F, Haffar S, Garg SK, Lake JR. Extra-hepatic manifestations associated with hepatitis $\mathrm{E}$ virus infection: a comprehensive review of the literature. Gastroenterol Rep (Oxf) 2016;4:1-15.

66. Serratrice J, Disdier P, Colson P, Ene N, de Roux CS, Weiller PJ. Acute polyarthritis revealing hepatitis E. Clin Rheumatol 2007;26:1973-1975.

67. Bialé L, Lecoules S, Galéano-Cassaz C, Carmoi T, Algayres JP. [Inflammatory polyarthralgia reveling acute hepatitis E]. Presse Med 2013;42:365-367.

68. Al-Shukri I, Davidson E, Tan A, et al. Rash and arthralgia caused by hepatitis E. Lancet 2013;382:1856.

69. Bazerbachi F, Leise MD, Watt KD, Murad MH, Prokop LJ, Haffar S. Systematic review of mixed cryoglobulinemia associated with hepatitis E virus infection: association or causation?
Gastroenterology Rep (Oxf) 2017;5:178-184.

70. Kamar N, Weclawiak H, Guilbeau-Frugier C, et al. Hepatitis E virus and the kidney in solid-organ transplant patients. Transplantation 2012;93:617-623.

71. Pischke S, Behrendt P, Manns MP, Wedemeyer H. HEV-associated cryoglobulinaemia and extrahepatic manifestations of hepatitis E. Lancet Infect Dis 2014;14:678-679.

72. Czaja AJ. Autoantibodies in autoimmune liver disease. Adv Clin Chem 2005;40:127-164.

73. Czaja AJ. Autoimmune liver disease and rheumatic manifestations. Curr Opin Rheumatol 2007;19:74-80.

74. Selmi C, Generali E, Gershwin ME. Rheumatic manifestations in autoimmune liver disease. Rheum Dis Clin North Am 2018;44:65-87.

75. Czaja AJ, Carpenter HA. Distinctive clinical phenotype and treatment outcome of type 1 autoimmune hepatitis in the elderly. Hepatology 2006;43:532-538.

76. Matsumoto T, Morizane T, Aoki Y, et al. Autoimmune hepatitis in primary Sjogren's syndrome: pathological study of the livers and labial salivary glands in 17 patients with primary Sjogren's syndrome. Pathol Int 2005;55:70-76.

77. Lis-Swiety A, Brzezinska-Wcislo L, Pierzchala E, WcisloDziadecka D. Systemic sclerosis-polymyositis overlap syndrome accompanied by autoimmune hepatitis and sarcoidosis of mediastinal lymphnodes. J Eur Acad Dermatol Venereol 2006;20:107-108.

78. Fusconi M, Vannini A, Dall'Aglio AC, et al. Anti-cyclic citrullinated peptide antibodies in type 1 autoimmune hepatitis. Aliment Pharmacol Ther 2005;22:951-955.

79. Montano-Loza A, Czaja AJ, Carpenter HA, et al. Frequency and significance of antibodies to cyclic citrullinated peptide in type 1 autoimmune hepatitis. Autoimmunity 2006;39:341-348.

80. Liberal R, Grant CR, Mieli-Vergani G, Vergani D. Autoimmune hepatitis: a comprehensive review. J Autoimmun 2013;41:126-139.

81. Fernández-Solà J. Management of extrahepatic manifestations in alcoholic liver disease. Clin Liver Dis (Hoboken) 2013;2:89-91.

82. López-Larramona G, Lucendo AJ, González-Castillo S, Tenias JM. Hepatic osteodystrophy: an important matter for consideration in chronic liver disease. World J Hepatol 2011;3:300-307.

83. Kizilgul M, Ozcelik O, Delibasi T. Bone health and vitamin D status in alcoholic liver disease. Indian J Gastroenterol 2016;35:253-259.

84. González-Reimers E, Quintero-Platt G, Rodríguez-Rodríguez E, Martínez-Riera A, Alvisa-Negrín J, Santolaria-Fernández F. Bone changes in alcoholic liver disease. World J Hepatol 2015;7:1258-1264.

85. López-Larramona G, Lucendo AJ, González-Delgado L. Alcoholic liver disease and changes in bone mineral density. Rev Esp Enferm Dig 2013;105:609-621.

86. Bang CS, Shin IS, Lee SW, et al. Osteoporosis and bone fractures in alcoholic liver disease: a meta-analysis. World J Gastroenterol 2015;21:4038-4047.

87. Eshraghian A. Bone metabolism in non-alcoholic fatty liver disease: vitamin D status and bone mineral density. Minerva Endocrinol 2017;42:164-172.

88. Kim G, Kim KJ, Rhee Y, Lim SK. Significant liver fibrosis assessed using liver transient elastography is independently associated with low bone mineral density in patients with non-alcoholic fatty liver disease. PLoS One 2017;12:e182202.

89. Lee SH, Yun JM, Kim SH, et al. Association between bone mineral density and nonalcoholic fatty liver disease in Korean adults. J Endocrinol Invest 2016;39:1329-1336.

90. Satapathy SK, Garg S, Chauhan R, et al. Beneficial effects of tumor necrosis factor-alpha inhibition by pentoxifylline on clinical, biochemical, and metabolic parameters of patients with nonalcoholic steatohepatitis. Am $J$ Gastroenterol 2004;99:1946-1952. 
91. Mazhar K. The future of nonalcoholic fatty liver disease treatment. Med Clin North Am 2019;103:57-69.

92. Hay JE. Bone disease in cholestatic liver disease. Gastroenterology 1995;108:276-283.

93. Floreani A, Cazzagon N. PBC and related extrahepatic diseases. Best Pract Res Clin Gastroenterol 2018;34-35:49-54.

94. Marí-Alfonso B, Simeón-Aznar CP, Guillén-Del Castillo A, et al; Systemic Autoimmune Diseases Study Group (GEAS). Hepatobiliary involvement in systemic sclerosis and the cutaneous subsets: Characteristics and survival of patients from the Spanish RESCLE Registry. Semin Arthritis Rheum 2018;47:849-857.
95. Faraawi R, Harth M, Kertesz A, Bell D. Arthritis in hemochromatosis. J Rheumatol 1993;20:448-452.

96. Husar-Memmer E, Stadlmayr A, Datz C, Zwerina J. HFE-related hemochromatosis: an update for the rheumatologist. Curr Rheumatol Rep 2014;16:393.

97. Martinon F, Pétrilli V, Mayor A, Tardivel A, Tschopp J. Goutassociated uric acid crystals activate the NALP3 inflammasome. Nature 2006;440:237-241.

98. McGonagle D, Tan AL, Madden J, Emery P, McDermott MF. Successful treatment of resistant pseudogout with anakinra. Arthritis Rheum 2008;58:631-633. 